\title{
Assessment of the Effect of 24-Hour Aldosterone Administration on Protein Abundance in Fluorescence-Sorted Mouse Distal Renal Tubules by Mass Spectrometry
}

\author{
Thomas B. Jensen ${ }^{a}$ Trairak Pisitkun ${ }^{c}$ Jason D. Hoffert ${ }^{c}$ Uffe B. Jensen ${ }^{a, b}$ \\ Robert A. Fenton ${ }^{a}$ Helle A. Praetorius ${ }^{a}$ Mark A. Knepper ${ }^{c}$ Jeppe Praetorius ${ }^{a}$ \\ a Department of Biomedicine and the Water and Salt Research Center, Aarhus University - Health, and \\ ${ }^{b}$ Institute of Clinical Medicine, Department of Clinical Genetics, Aarhus University Hospital, Aarhus, Denmark; \\ 'Epithelial Systems Biology Laboratory, National Heart, Lung and Blood Institute, National Institutes of Health, \\ Bethesda, Md., USA
}

\section{Key Words}

Isobaric tags for relative and absolute quantitation .

Fluorescence-activated cell sorting · Mass spectrometry .

Aldosterone

\begin{abstract}
Background/Aims: Aldosterone exerts multiple long-term effects on the distal renal tubules. The aim of this study was to establish a method for identifying proteins in these tubules that change in abundance by only 24 -hour aldosterone administration. Methods: Mice endogenously expressing green fluorescent protein (eGFP) in the connecting tubule and cortical collecting ducts were treated with a subcutaneous injection of $2.0 \mathrm{mg} / \mathrm{kg}$ aldosterone or vehicle $(\mathrm{n}=5)$, and sacrificed $24 \mathrm{~h}$ later. Suspensions of single cells were obtained enzymatically, and eGFP-positive cells were isolated by fluorescence-activated cell sorting (FACS). Samples of $100 \mu \mathrm{g}$ of proteins were digested with trypsin and labeled with 8-plex isobaric tags for relative and absolute quantitation reagents and processed for liquid chromatography-tandem mass spectrometry (LC-MS/MS). Results: FACS yielded 1.4 million cells per mouse. The LC-MS/MS
\end{abstract}

spectra were matched to peptides by the SEQUEST search algorithm, which identified 3,002 peptides corresponding to 506 unique proteins, of which 20 significantly changed abundance $24 \mathrm{~h}$ after aldosterone injection. Conclusion: We find the method suitable and useful for studying hormonal effects on protein abundance in distal tubular segments.

Copyright $\odot 2013$ S. Karger AG, Basel

\section{Introduction}

The principal cells in the late distal convoluted tubule (DCT2), the connecting tubule (CNT), and the initial cortical collecting duct (iCCD) play a critical role in finetuning the $\mathrm{Na}^{+}$reabsorption, $\mathrm{K}^{+}$secretion and total body fluid volume [1]. Here, aldosterone is intricately involved in this regulation, and is produced in the cortex of the adrenal gland in response to elevated plasma concentrations of angiotensin II or $\mathrm{K}^{+}[1]$.

Hypothesis-driven studies have so far successfully dealt with aldosterone's effects on known transport proteins or their regulatory pathways in renal tubules. The aim of this study was to establish a method for more broadly identify-

\section{KARGER}

Fax +4161306 1234

E-Mail karger@karger.ch

www.karger.com
(C) 2013 S. Karger AG, Basel

$1660-2137 / 12 / 1214-0009 \$ 38.00 / 0$

Accessible online at:

www.karger.com/nep
Jeppe Praetorius

Department of Biomedicine, Aarhus University - Health

Wilhelm Meyers Allé 3, Building 1233/1234

DK-8000 Aarhus C (Denmark)

E-Mail jp@ana.au.dk 
ing proteins in the principal cells of the DCT2, CNT and iCCD that are regulated $24 \mathrm{~h}$ after aldosterone administration. Current large-scale mass spectrometry-based proteomic analysis offers a way to identify and quantify a large amount of proteins in a certain cell type with high accuracy and speed [2]. Liquid chromatography-tandem mass spectrometry (LC-MS/MS) uses mass to charge ratio to identify peptides in a solution. We utilized an approach to first tag each peptide sample with isobaric tags for relative and absolute quantitation (iTRAQ) molecules. The quantitation is done on the iTRAQ reporter ion intensities instead of the mass to charge spectra. The advantages of iTRAQ include the ability to run the samples simultaneously. However, such analysis requires a relatively large amount of protein, commonly up to $100 \mu \mathrm{g}$ or more per sample. Previously, the yield of protein obtained from manually isolated renal tubules has been quite low and hindered large-scale proteomic studies.

We exploited a transgenic mouse line endogenously expressing enhanced green fluorescent protein (eGFP) in the late DCT, CNT and iCCD to sort renal cells based on their fluorescent properties and obtain enriched populations of cells from these tubules. We identified more than 500 proteins from the DCT, CNT and iCCD, and the abundance of several of these novel proteins was affected by aldosterone administration.

\section{Methods}

\section{Animals and Aldosterone Administration}

Transgenic c57bl/ 6 mice expressing eGFP driven by the TRPv5 promoter in DCT2, CNT and iCCD [3] were injected subcutaneously with $2.0 \mathrm{mg} / \mathrm{kg}$ aldosterone (Sigma-Aldrich) in sunflower seed oil or vehicle $24 \mathrm{~h}$ prior to sacrifice. Male c57bl/6 mice (Taconic) were treated similarly with an extra group receiving two $2.0 \mathrm{mg} /$ $\mathrm{kg}$ aldosterone injections 24 and $48 \mathrm{~h}$ before sacrifice for immunostaining. Plasma aldosterone was assessed by Coat-a-Count Aldosterone Kit (Siemens). The authors are licensed to breed the GMO mice and conduct the described experiments by the Danish Ministry of Justice.

\section{Cell Isolation and Fluorescence-Activated Cell Sorting}

Blood was drawn during isofluorane anesthesia, and kidneys were perfused with $37^{\circ} \mathrm{C}$ physiological isolation solution with $1 \mu \mathrm{l} /$ $\mathrm{ml}$ proteinase K (Roche Diagnostics) [3]. Each kidney was sliced and incubated for $45 \mathrm{~min}$ in isolation solution on a shaking table at $37^{\circ} \mathrm{C}$. Tubules were digested to single cells using a trypsin/ EDTA solution (Invitrogen) twice at $37^{\circ} \mathrm{C}$ for $5 \mathrm{~min}$. The cells were passed through a $40-\mu \mathrm{m}$ mesh, and eGFP-positive kidney cells were isolated on a FACSAria III (BD Biosciences). Dead cells were excluded by propidium iodide uptake. Isolates were added to $8 \mathrm{M}$ urea buffer and sonicated on ice. The lysate was centrifuged at $10,000 \mathrm{~g}$, and the supernatant was stored at $-80^{\circ} \mathrm{C}$.

\section{Labeling with iTRAQ}

Samples were pooled to obtain $100 \mu \mathrm{g}$ of protein each. A total of 3 aldosterone-treated samples and 3 vehicle-treated samples were obtained from 5 mice in each group. Two whole kidney homogenates were used as control of enrichment. Eight-plex iTRAQ labeling (Applied Biosystems) tagged control samples 1-3 with iTRAQ molecules 113-115, respectively, aldosterone samples 1-3 with 116-118, and the whole kidney homogenates with 119 and 121 and processed as previously described [4].

\section{Liquid Chromatography-Tandem Mass Spectrometry}

Samples were analyzed by LC-MS/MS using an Eksigent nanoflow LC system connected to an LTQ Orbitrap Velos mass spectrometer (Thermo Scientific) as described previously [4]. MS2 spectra were used for peptide identification by running the SEQUEST algorithm (Proteome Discoverer 1.2, Thermo Scientific). Peptides that matched multiple proteins were identified using ProMatch software and excluded from further analysis.

Tissue Fixation and Immunohistochemical Staining

Kidneys were perfusion fixed and stained as described previously [3]. Here, sections were incubated overnight with rabbit anti-pyruvate kinase antibody (PKM2, Epitomics). Fluorescence imaging was performed on a Leica DM IRE2 confocal microscope and semi-quantitation was performed as previously described [5].

\section{Statistics}

For quantification of MS data, reporter ion peak intensity for peptides belonging to the same protein were summed for each sample and tested by two-tailed t tests choosing a significance level of $\mathrm{p}<0.05$.

\section{Results}

\section{Validation of Plasma Aldosterone Increase}

The plasma aldosterone concentration after $24 \mathrm{~h}$ of treatment increased more than 5-fold compared to the vehicle-treated group (fig. 1a). The normal value is $20 \mathrm{ng} /$ $\mathrm{dl}$ [6]. Plasma $\mathrm{K}^{+}$was $3.56 \pm 0.17 \mathrm{~mm}$ in vehicle-treated and $2.75 \pm 0.16 \mathrm{mM}$ at $24 \mathrm{~h}$ after aldosterone administration $(\mathrm{p}<0.01, \mathrm{n}=6)$. Thus, it seems that a suitable increase in plasma aldosterone concentration was achieved within the 24-hour timeframe by the chosen protocol.

\section{Enzymatic Digestion and Isolation of eGFP-Positive Cells}

Enrichment of the samples with DCT2, CNT and iCCD cells and minimizing other renal cells was achieved by (1) exploiting a mouse line with endogenous expression of eGFP in DCT2, CNT and iCCD (fig. 1b); (2) enzymatic digestion of kidneys into single tubules (fig. 1c), and (3) single cells (fig. 1d) of which a minority were eGFP positive (fig. 1e). Subsequent fluorescence-activated cell sorting (FACS; fig. 2a) separated eGFP-expressing 

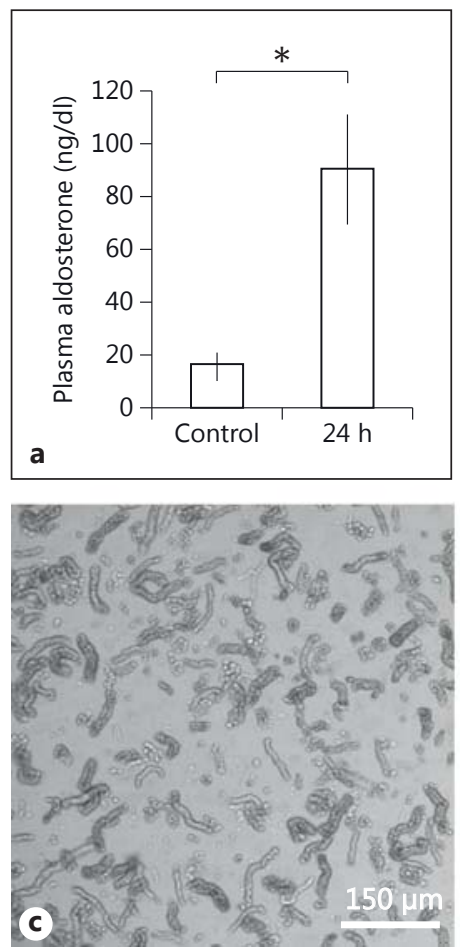

Fig. 1. Aldosterone administration and production of single cell suspensions. a Plasma aldosterone levels in vehicle-treated (control) and aldosterone-treated mice $(24 \mathrm{~h}, \mathrm{p}<0.01, \mathrm{n}=5)$. b Schematic representation of the renal tubular system. The green color indicates tubular segments expressing eGFP driven by the TRPv5 promoter in the transgenic mouse line. c Differential interference
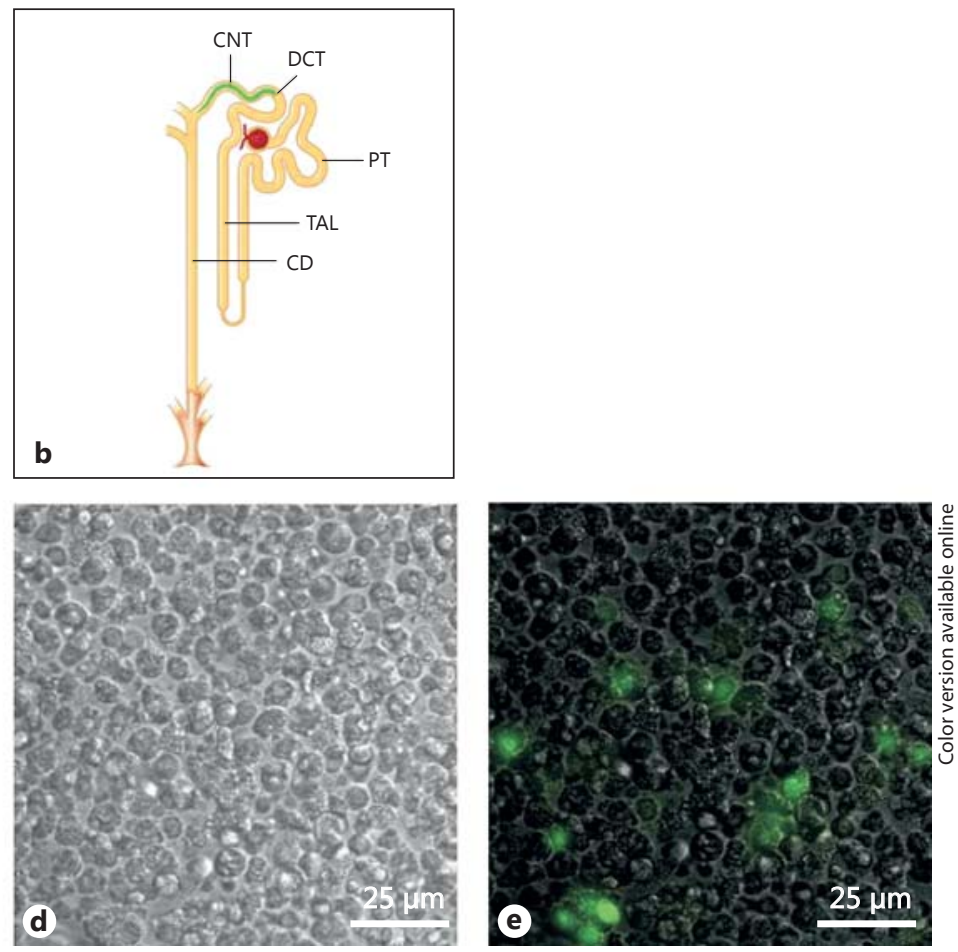

contrast micrograph of the enzymatically detached renal cortical tubules. d Differential interference contrast micrograph exemplifying the enzymatically detached renal cortical cells. e The same contrast image with an overlay of the endogenous eGFP fluorescence. Colors refer to the online version only.

tive phosphorylation. Table 1 lists common markers of DCT2/CNT/iCCD and other proteins that were enriched by the FACS compared to whole kidney and markers from other tubules that are found in lower abundance after FACS. No markers of glomeruli, proximal tubules, thin limbs or thick ascending limbs were enriched by the procedure.

\section{Identification of Aldosterone-Regulated Polypeptides}

Stringent criteria were set for the MS2 spectra based on the peptide elution profile and reporter ion intensities, allowing only peptides that had been identified in all the samples to be included in the quantitation. A total of 3,002 peptides from 506 unique proteins were identified by MS, and of these 1,455 peptides were quantified, corresponding to 289 proteins. Twenty of these proteins were significantly regulated by aldosterone. Figure 3 shows FACS-enriched proteins with significantly increased or decreased abundance at $24 \mathrm{~h}$ after aldosterone administration in the MS2 run, while figure 4 shows the aldosterone-regulated proteins that were not enriched by FACS. 


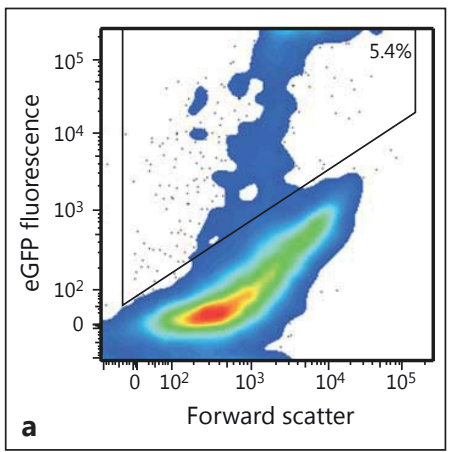

Fig. 2. Fluorescence-activated isolation of single eGFP-positive cells. a Scatter diagram of eGFP fluorescence intensity as a function of the forward scatter. The boxed area indicates the fraction of cells chosen for FACS. b Similar scatter diagram showing the reanalysis

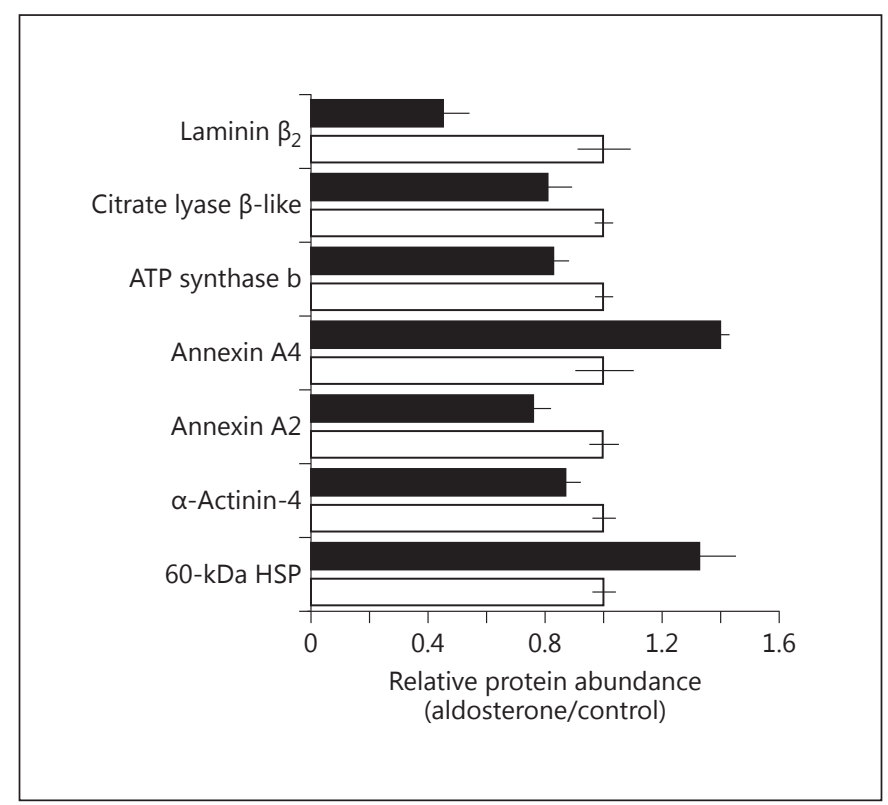

Fig. 3. FACS-enriched proteins regulated by 24 -hour aldosterone administration. Mass spectrometry identified a number of proteins, which were enriched by the isolation on eGFP-positive cells. The abundance of some of these proteins was significantly changed $24 \mathrm{~h}$ after aldosterone administration $(\mathrm{p}<0.05)$. The graph shows the mean \pm SEM protein abundance after aldosterone administration relative to the control values. For normalization of the MS data, all peptide intensities were divided by the median intensity for the sample. Statistical analysis was based on both the individual peptide ratios and the summed peptide peak intensities for a given protein. HSP $=$ Heat shock protein; ATP synthase $b=$ ATP synthase subunit b; citrate lyase $\beta$-like $=$ citrate lyase subunit $\beta$-like protein; laminin $\beta_{2}=$ laminin subunit $\beta_{2}$.
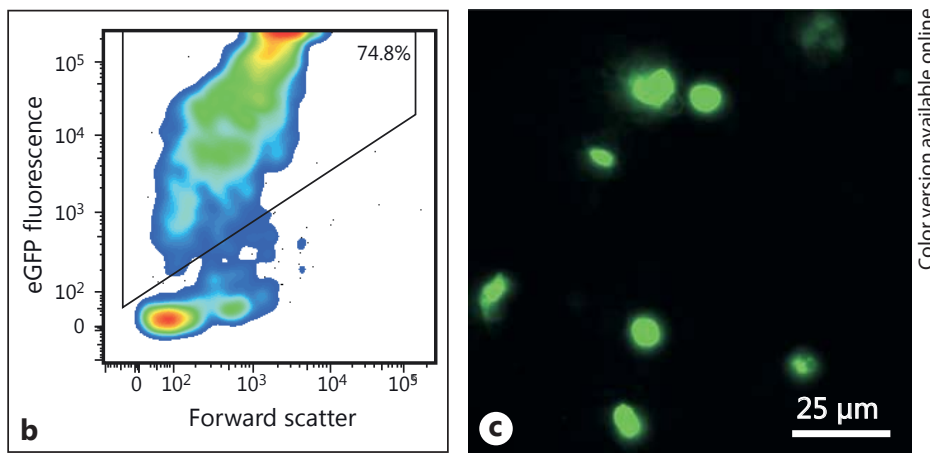

of sorted eGFP cells. Numbers indicate percentage of cells in the boxed region of interest. c Fluorescence micrograph of the living, sorted eGFP-positive cells resulting from FACS.

Table 1. Proteins enriched or diminished in eGFP cells after FACS compared to whole kidney homogenate

\begin{tabular}{llll}
\hline Enriched & Log2 & Diminished & Log2 \\
\hline Aquaporin 2 & 2.06 & Albumin & -2.04 \\
$11 \beta$-dehydrogenase 2 & 1.79 & Villin-1 & -1.55 \\
Calbindin 1 & 1.76 & NaPi2 & -1.09 \\
Green fluorescent protein & 1.38 & Clathrin & -0.70 \\
Pyruvate dehydrogenase E1a & 1.31 & Lrp2/megalin & -0.60 \\
Kallikrein 1 & 1.08 & NHE-RF1 & -0.38 \\
Sodium chloride cotransporter & 0.43 & Ezrin & -0.22 \\
\hline
\end{tabular}

Numbers are the $\log 2$ to the ratios of the protein abundance in GFP cells relative to the homogenate $[\log 2$ (eGFP cells/homogenate)] as determined by LC-MS/MS.

$\mathrm{NaPi} 2=$ Sodium phosphate cotransporter 2; NHE-RF1 $=\mathrm{Na}^{+} /$ $\mathrm{H}^{+}$exchange regulatory cofactor.

Validation of Selected Aldosterone-Regulated Proteins

Several proteins changed numerically in abundance upon aldosterone administration without reaching statistical significance, with a trend towards increases in glycolytic enzymes and a decrease in glyconeogenic enzymes. To assess whether these enzymes were in fact increased by other methods, one enzyme with an apparently large change in abundance $(60 \%)$ was selected for further analysis: pyruvate kinase, the rate-limiting step in the glycolysis. Immunohistochemical analysis was performed to verify the regulation of pyruvate kinase in separate experiments on wild-type mice. The antibody against the pyruvate kinase M2 isoform in the TRPv5- 


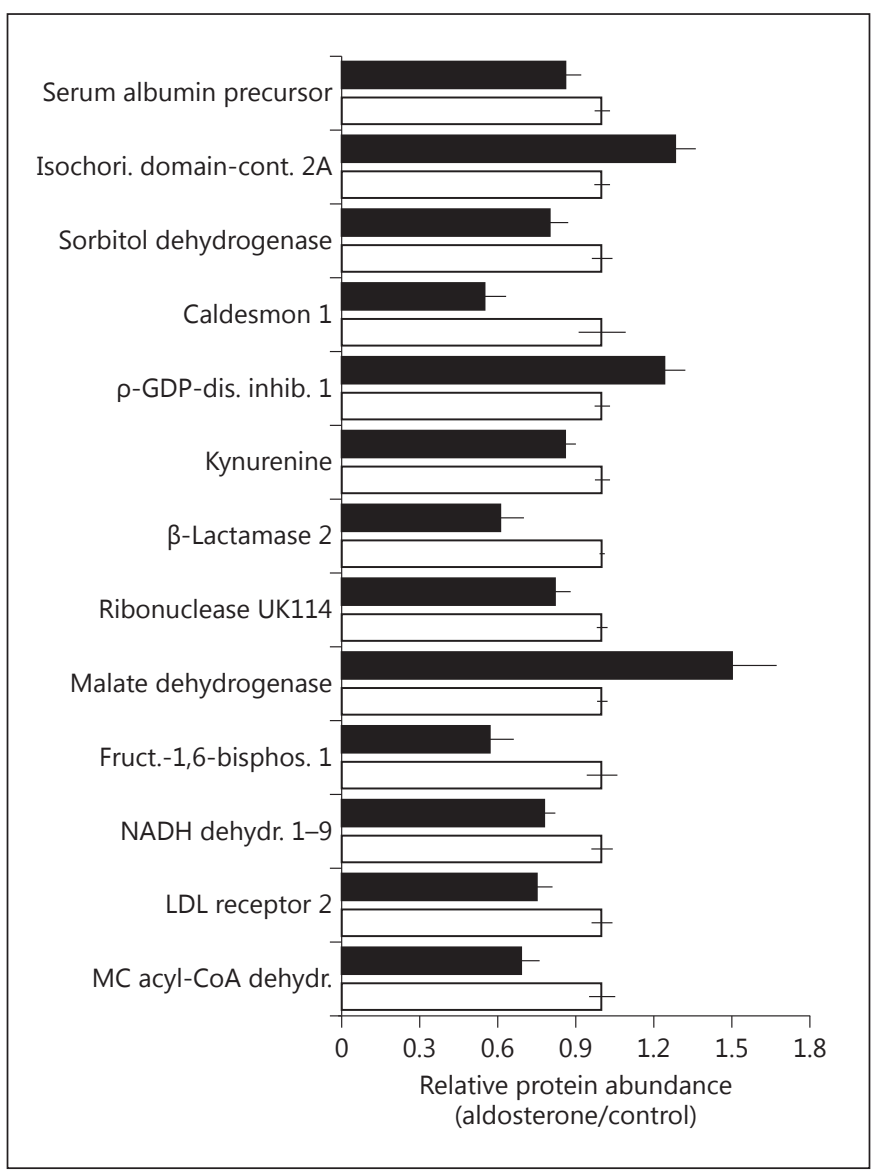

Fig. 4. Non-enriched proteins regulated by 24-hour aldosterone administration. Mass spectrometry identified a number of aldosterone-regulated proteins $(\mathrm{p}<0.05)$, which were not enriched by the isolation on eGFP-positive cells. The graph shows the mean \pm SEM protein abundance after aldosterone administration relative to the control values. MC acyl-CoA dehydr. = Medium-chain specific acyl-CoA dehydrogenase; LDL receptor $2=$ low-density lipoprotein receptor-related protein 2; NADH dehydr. 1-9 = NADH dehydrogenase (ubiquinone)-1a subcomplex subunit 9; fruct.-1,6bisphos. 1 = fructose-1,6-bisphosphatase $1 ; \beta$-lactamase $2=\beta$ lactamase-like protein 2 ; kynurenine $=$ kynurenine $/ \alpha$-aminoadipate aminotransferase; $\rho$-GDP dis. inhib. $1=\rho$-GDP dissociation inhibitor 1 ; isochori. domain-cont. $2 \mathrm{~A}=$ isochorismatase domaincontaining protein $2 \mathrm{~A}$.

positive tubules selectively stained near the apical domain of these tubules and seemed to be regulated by aldosterone administration (fig. 5a). Pyruvate kinase M2 abundance was increased by $77 \%$ at $24 \mathrm{~h}$, and by $190 \%$ at $48 \mathrm{~h}$ after aldosterone administration compared to controls as assessed by semiquantitative immunofluorescence histochemistry $(\mathrm{p}>0.05, \mathrm{p}<0.05$, respectively, $n=6$; fig. $5 b)$.

Early Aldosterone-Induced Proteins in the Distal Tubules

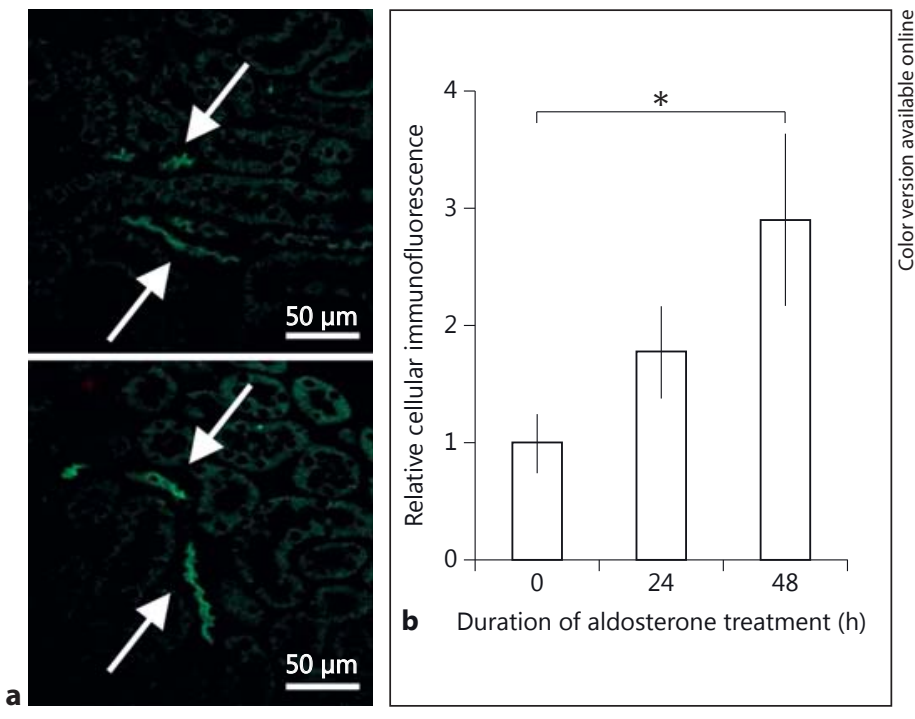

Fig. 5. Regulation of renal pyruvate kinase abundance by aldosterone. Immunohistochemical analysis of kidney sections was performed for pyruvate kinase. a Representative immunostaining with an antibody against the pyruvate kinase M2 isoform in the TRPv5-positive tubule in control kidneys (top panel) and in a kidney $48 \mathrm{~h}$ after aldosterone administration (bottom panel). Arrows mark these segments. b Pyruvate kinase abundance semi-quantified from similar micrographs after correction for background, cell area and normalization to control values $(n=6)$.

\section{Discussion}

In this study, we present a method to evaluate hormonal effects on protein abundance in DCT2, CNT and iCCD, and report the short-term effects of aldosterone in these TRPv5-positive tubules. FACS was chosen for the isolation of renal cells because of its advantageous speed and yield in order to gain enough viable material for quantitative mass spectrometry. The highest purity achieved in the current study was $79 \%$. This is probably due to the complex mixture of cells and debris derived from minced and enzymatically digested kidney including matrix proteins as compared to samples isolated from e.g. blood. To minimize contamination with cell debris, FACS was optimized for excluding small particles. In a similar study by Da Silva et al. [7], the yield was much smaller but of higher purity.

Although some membrane proteins would be expected to be cleaved by the enzymatic treatment, a following study showed no change in migration of AQP2 of the $\mathrm{Na}^{+}$, $\mathrm{K}^{+}$-ATPase in isolated cells by immunoblotting [Poulsen and Christensen, pers. commun.]. Only sodium chloride 
cotransporter (NCC) migrated as a single band of approximately $70 \mathrm{kDa}$ instead of $140 \mathrm{kDa}$. These observations and the lack of cell 'blebbing' on routine microscopical inspection indicate that the isolated cells and even the proteins are in a state fit for further analysis. By including whole renal cortex samples in the iTRAQ, we have validated the FACS enrichment of typical distal tubules such as AQP2, NCC, corticosteroid $11 \beta$-dehydrogenase 2 , kallikrein-1, and calbindin, while contaminating proteins from other tubular segments were found in lower abundance.

Applying our stringent criteria could potentially mask most actual changes between samples. For instance, one identified protein enriched in our eGFP-positive cells aquaporin 2 has a ratio of more than 2, but was excluded for quantification by the software algorithm based on peptide elution profile and reporter ion intensities. The iTRAQ technique has a known 'ratio compression' problem (i.e. reporter ions from coeluted contaminant peptides dilute out the real changes leading to a compression of ratios toward 1:1). This problem in general makes quantification less significant, and currently there is no well-accepted way to solve this. In this study, the abundance of the thiazide-sensitive NCC, a well-known target for aldosterone in the DCT [8], increased by only $10 \%$ in the aldosterone-treated group $(\mathrm{p}=0.21)$ compared to controls. Using a cutoff based on a fold change only (e.g. $>1.5$ or $<0.67$ ), the linked database provides several additional candidate proteins that would be worth pursuing for further validation.

We present seven FACS-enriched proteins with significantly changed abundance $24 \mathrm{~h}$ after aldosterone administration of very diverse cellular functions (fig. 3). Interestingly, annexins 2 and 4 are cytosolic phospholipidbinding proteins involved in organizing exocytosis or endocytosis [9]. The change in abundance for both proteins by hormonal status has been documented in the renal tubules previously [10]. Also laminin subunit- $\beta_{2}$ is known to have a restricted tissue distribution, which includes the collecting ducts [11].

Aldosterone is known to increase $\mathrm{Na}^{+}$reabsorption and $\mathrm{K}^{+}$secretion by enhancing basolateral $\mathrm{Na}^{+}, \mathrm{K}^{+}-$ ATPase activity [12], apical $\mathrm{Na}^{+}$influx through $\mathrm{ENaC}$ [13] and the apical $\mathrm{K}^{+}$exit through ROMK channels [14, 15]. The increased need for ATP to drive the $\mathrm{Na}^{+}, \mathrm{K}^{+}-$ ATPase is met by an aldosterone-induced increase in the activity of the respiratory chain enzymes [16] and enzymes in mitochondrial ATP synthesis [17]. Pyruvate kinase is one rate-limiting enzyme in the glycolysis; it served in this study as an example of a protein with a relatively large change in abundance that did not reach statistical significance by MS2 but did so by semiquantitative immunohistochemistry. Handler et al. [16] showed that aldosterone administration decreased the pyruvate kinase substrate phosphoenolpyruvate and increased pyruvate in toad bladder implying an increased enzyme activity. We show that this may indeed also be the case in the CNT and collecting duct, which are the mammal equivalents of the toad bladder.

In conclusion, we present a method to isolate viable mouse DCT2, CNT and iCCD cells using endogenous eGFP and FACS. Mass spectrometry identified 20 proteins that were significantly regulated in abundance $24 \mathrm{~h}$ after aldosterone administration, and we provide a database containing many more proteins that are potentially regulated. It is our hope that further analysis of the database may spur new investigations of the early aldosterone-regulated proteins in renal tubules.

\section{Acknowledgements}

We wish to thank Inger Merete S. Paulsen, Else-Merete Løcke, Helle Høyer and Christian V. Westberg for expert technical assistance. We are grateful for the help and input from Charlotte Christie Petersen, Anni Skovbo, Anette Thomsen from the FACS core facility, Health, Aarhus University. We would like to thank Marjan Gucek and Guanghui Wang from the NHLBI Proteomics Core Facility, NIH for running the LC-MS/MS.

Financial support was granted from The Danish Council for Independent Research Medical Sciences, the Lundbeck Foundation, and Danish Heart Association. The Water and Salt Research Center at Aarhus University was established and supported by the Danish National Research Foundation.

\section{Disclosure Statement}

The authors declare no conflicts of interest.

References

1 Boron WF, Boulpaep EL (eds): Medical Physiology: A Cellular and Molecular Approach. Saunders/Elsevier, Philadelphia, 2009.

$\checkmark 2$ Walther TC, Mann M: Mass spectrometrybased proteomics in cell biology. J Cell Biol 2010;190:491-500.

-3 Hofmeister MV, Fenton RA, Praetorius J: Fluorescence isolation of mouse late distal convoluted tubules and connecting tubules: effects of vasopressin and vitamin $\mathrm{D}_{3}$ on $\mathrm{Ca}^{2+}$ signaling. Am J Physiol Renal Physiol 2009; 296:F194-F203. 
4 Hoffert JD, Pisitkun T, Saeed F, Song JH, Chou CL, Knepper MA: Dynamics of the G protein-coupled vasopressin V2 receptor signaling network revealed by quantitative phosphoproteomics. Mol Cell Proteomics 2012; 11:M111.014613.

5 Damkier HH, Praetorius J: Genetic ablation of Slc4a10 alters the expression pattern of transporters involved in solute movement in the mouse choroid plexus. Am J Physiol Cell Physiol 2012;302:C1452-C1459.

-6 Arrighi I, Bloch-Faure M, Grahammer F, Bleich M, Warth R, Mengual R, Drici MD, Barhanin J, Meneton P: Altered potassium balance and aldosterone secretion in a mouse model of human congenital long QT syndrome. Proc Natl Acad Sci USA 2001;98: 8792-8797.

-7 Da Silva N, Pisitkun T, Belleannee C, Miller LR, Nelson R, Knepper MA, Brown D, Breton $\mathrm{S}$ : Proteomic analysis of V-ATPase-rich cells harvested from the kidney and epididymis by fluorescence-activated cell sorting. Am J Physiol Cell Physiol 2010;298:C1326-C1342.
8 van der Lubbe N, Lim CH, Meima ME, van Veghel R, Rosenbaek LL, Mutig K, Danser $\mathrm{AH}$, Fenton RA, Zietse R, Hoorn EJ: Aldosterone does not require angiotensin II to activate NCC through a WNK4-SPAK-dependent pathway. Pflugers Arch 2012;463:853863.

-9 Carr G, Simmons NL, Sayer JA: Disruption of clc-5 leads to a redistribution of annexin A2 and promotes calcium crystal agglomeration in collecting duct epithelial cells. Cell Mol Life Sci 2006;63:367-377.

10 Hoorn EJ, Hoffert JD, Knepper MA: Combined proteomics and pathways analysis of collecting duct reveals a protein regulatory network activated in vasopressin escape. J Am Soc Nephrol 2005;16:2852-2863.

11 Yoshida T, Muller E, Stears R, Shirota S, Tsuchiya K, Akiba T, Gullans SR: Osmoadaptation-related genes in inner medulla of mouse kidney using microarray. Biochem Biophys Res Commun 2004;322:250-257.

12 Palmer LG, Antonian L, Frindt G: Regulation of the Na-K pump of the rat cortical collecting tubule by aldosterone. J Gen Physiol 1993; 102:43-57.
13 Muller OG, Parnova RG, Centeno G, Rossier BC, Firsov D, Horisberger JD: Mineralocorticoid effects in the kidney: correlation between alphaENaC, GILZ, and Sgk-1 mRNA expression and urinary excretion of $\mathrm{Na}^{+}$and $\mathrm{K}^{+}$. J Am Soc Nephrol 2003;14:1107-1115.

14 Barger AC, Berlin RD, Tulenko JF: Infusion of aldosterone, 9-alpha-fluorohydrocortisone and antidiuretic hormone into the renal artery of normal and adrenalectomized, unanesthetized dogs: effect on electrolyte and water excretion. Endocrinol 1958;62:804815.

15 Palmer LG, Choe H, Frindt G: Is the secretory $\mathrm{K}$ channel in the rat CCT ROMK? Am J Physiol Renal Physiol 1997;273:F404-F410.

16 Handler JS, Preston AS, Orloff J: The effect of aldosterone of glycolysis in the urinary bladder of the toad. J Biol Chem 1969;244:31943199.

17 Sharp GW, Leaf A: Metabolic requirements for active sodium transport stimulated by aldosterone. J Biol Chem 1965;240:4816-4821. 